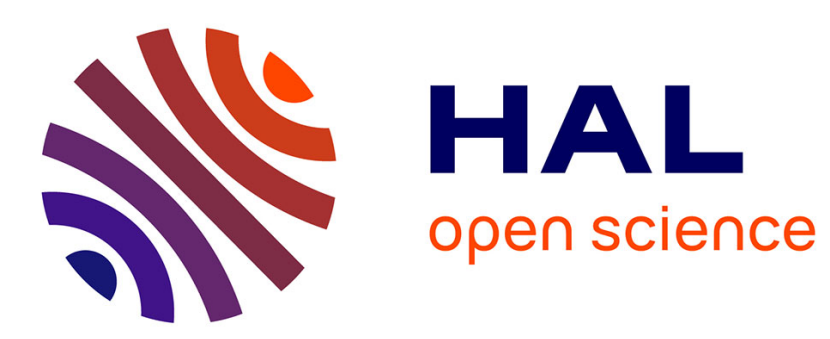

\title{
Enquête sur l'éducation en milieu rural et en milieu urbain
}

\author{
Gaston Lanneau, Philippe Malrieu
}

\section{To cite this version:}

Gaston Lanneau, Philippe Malrieu. Enquête sur l'éducation en milieu rural et en milieu urbain. Enfance, 1957, nº 4 , pp. 465-482. halshs-01205997

\section{HAL Id: halshs-01205997 https://shs.hal.science/halshs-01205997}

Submitted on 28 Sep 2015

HAL is a multi-disciplinary open access archive for the deposit and dissemination of scientific research documents, whether they are published or not. The documents may come from teaching and research institutions in France or abroad, or from public or private research centers.
L'archive ouverte pluridisciplinaire HAL, est destinée au dépôt et à la diffusion de documents scientifiques de niveau recherche, publiés ou non, émanant des établissements d'enseignement et de recherche français ou étrangers, des laboratoires publics ou privés. 


\title{
ENQUÊTE SUR L'ÉDUCATION EN MILIEU RURAL ET EN MILIEU URBAIN
}

\author{
Gaston LANNEAU et Philippe MALRIEU
}

Enfance, $\mathrm{N}^{\circ} 4,1957,465-482$

\begin{tabular}{|lll|}
\hline & \multicolumn{2}{c|}{ MOTS CLÉS } \\
\hline École mixte & Jeux & Personnalisation \\
Éducation morale & Maîtrise du corps & Socialisation \\
Hétéronomie & Milieu rural & Vie sociale \\
\hline
\end{tabular}

\section{RÉSUMÉ}

Enquête dans la région toulousaine sur les comportements éducatifs (observation des parents dans leurs rapports avec les enfants ; examen des comportements de l'enfant, entretien avec les parents sur leurs conceptions pédagogiques).

Un examen psychologique de l'enfant est fait au point de vue caractériel et intellectuel. Il vise à définir l'allure des réactions motrices, l'aptitude à fixer les habitudes, l'émotivité, la structure des réactions intellectuelles, les résultats scolaires et l'attitude en classe.

L'éducation à la campagne est plus homogène, d'une famille à l'autre, qu'elle ne l'est à la ville. Elle se caractérise par la lenteur des processus de socialisation : langage tardivement acquis, jeux collectifs souvent restreints au groupe des frères et sours, et souvent concurrencés par le travail, contacts culturels réduits, ce qui n'est guère propice à la formation d'une personnalité diversifiée, aux réactions sociales souples et riches. L'enfant est happé par la vie familiale, il vit pour elle, en fonction d'elle et dans un milieu homogène par sa composition sociale, amorphe dans sa structure.

La famille fournit les valeurs, les idéaux qui occupent les pensées de l'enfant : travail, production, propriété. C'est en les adoptant que l'enfant a conscience de grandir et de devenir un homme, puisqu'il n'y a pas de groupements extra familiaux, qui apporteraient à l'adolescent la conscience qu'il peut remplir une multiplicité de rôles.
\end{abstract}




\section{Enquête sur l'éducation en milieu rural et en milieu urbain}

Les consultations de parents mettent en évidence la multiplicité des conceptions et des pratiques pédagogiques qui dominent dans les familles françaises : ce ne sont pas seulement les idéaux qui diffèrent, ce sont aussi les techniques, les attitudes, les croyances relatives au rôle de l'enfance. Est-il possible de déceler, audessous de cette diversité, quelques grands types pédagogiques, et de les rapporter à quelques facteurs primordiaux ? Est-il possible aussi de savoir comment la personnalité de l'enfant se trouve influencée par l'éducation qu'il reçoit?

Une enquête a été entreprise, dans la région toulousaine, en vue d'étudier ces deux problèmes. Elle s'efforce d'atteindre le comportement éducatif de trois côtés différents :

- par l'observation des parents dans leurs rapports avec les enfants ;

- par l'examen des comportements de l'enfant, en tant qu'ils semblent refléter les influences de l'éducation familiale ;

- par un entretien avec les parents sur leurs conceptions pédagogiques.

Un examen psychologique de l'enfant est fait au point de vue caractériel et intellectuel. Il vise à définir l'allure des réactions motrices, l'aptitude à fixer les habitudes, l'émotivité, la structure des réactions intellectuelles, les résultats scolaires et l'attitude en classe.

L'enquête s'ouvre par une description de la structure de la famille, de ses sources de revenus, de son logement, de ses intérêts, de ses relations, de ses loisirs, afin de saisir quelques caractéristiques sociales et culturelles de la vie du foyer.

Nous avons retenu, comme étant particulièrement précises, vingt-neuf monographies : douze d'entre elles concernent des familles paysannes de régions de polyculture (Aveyron d'une part, Lauragais d'autre part) ; il s'agit de petits propriétaires exploitants, pour la plupart peu fortunés ; dix-sept études concernent des familles urbaines (milieux ouvriers, classes. moyennes).

Pour si importantes que soient les différences entre la ville et la campagne, il nous a semblé que le comportement éducatif était constitué, dans tous les cas, par quatre types de préoccupations hétérogènes, très inégalement répartis dans les diverses familles, et qui sont susceptibles d'entrer en conflit, de provoquer entre le père et la mère, ou entre les parents et les grands parents, certaines divergences.

C'est d'abord l'élevage de l'enfant : les premiers soins au nourrisson, le souci d'assurer sa santé, sa croissance, de le protéger contre les accidents, de lui assurer une maîtrise élémentaire du milieu physique. Ici apparaît la conscience de l'originalité de l'enfance : de sa faiblesse, de ses besoins ; elle est plus ou moins claire : d'ordre affectif en général, elle peut devenir systématique dans certaines familles urbaines sous l'influence des théories pédagogiques de la spontanéité.

Un deuxième groupe de comportements se rapporte au souci de la discipline sociale. Il s'agit, notamment, du dressage des besoins, du refoulement de l'agressivité, de l'initiation à la politesse, au sens de la hiérarchie et des distances sociales. 
L'hétéronomie est ici prépondérante et l'éducateur agit en développant la peur de la punition, le désir de la récompense. Il obéit à des coutumes qu'il n'a généralement pas songé à discuter - mené, dans une grande mesure, par le souci de faire respecter son personnage d'adulte, responsable de l'enfant devant la société.

Un troisième groupe comprend les attitudes qui visent la formation du personnage social, du futur travailleur, du futur citoyen. On s'efforce de donner à l'enfant le sens de la collaboration, de la civilité librement consentie, de la gentillesse, de l'entraide. Il s'agit moins de leçons que d'habitudes lentement conférées à l'enfant. Mais les perspectives des éducateurs sont plus conscientes que pour les pratiques antérieures ; elles se fondent sur un réseau de croyances, discutées dans la famille, et même dans des cercles plus vastes. Les méthodes pédagogiques ne se bornent pas à celles du dressage : on expose à l'enfant les raisons des encouragements ou des réprimandes.

On peut enfin ranger dans une dernière catégorie tout ce qui relève de la pédagogie morale consciente, de l'éducation de la personne. Les comportements éducatifs deviennent ici des plus complexes et des plus divers. C'est d'une part l'éducation de la maîtrise de soi : persévérance, autonomie, vie sexuelle. C'est d'autre part tout ce qui concerne la pédagogie culturelle; le souci d'expliquer, l'attention portée à l'instruction de l'enfant, à l'éducation artistique. C'est l'effort pour développer l'altruisme. Et c'est, enfin, la somme des initiations morales, religieuses, sociales, politiques, philosophiques, qui découvrent à l'enfant les perspectives de la vie.

Cette classification est en un sens artificielle : il n'y a pas de coupure tranchée entre les comportements pédagogiques, et nous aurons précisément à apprécier leurs interactions. Ce qui la justifie cependant, c'est qu'en chacune des catégories, les motivations des parents sont d'origines différentes. Il s'agit pour la première d'une affection encore un peu vitale, sensuelle, éveillée par le spectacle des impuissances et de l'innocence du jeune enfant. Puis vient le besoin d'obtenir un accord plutôt grégaire, avec, parfois, un désir de domination sur la génération nouvelle et un peu ennemie, ou tout au moins un peu gênante par son impulsivité. Apparaît, simultanément, la préoccupation d'assurer l'adaptation des jeunes à la vie sociale et à ses lois : on veut préserver l'enfant des échecs scolaires et professionnels, la motivation est essentiellement pragmatique. Et c'est, enfin, le désir d'assurer à l'enfant, ou plutôt à l'homme à venir, l'autonomie, l'intégration à un type de civilisation plus ou moins librement choisi par les éducateurs.

II est rare que les parents prennent conscience de cet ensemble de motivations. En eux se mêlent des élans qui prennent leur source dans l'amour sexuel, des inquiétudes relatives à l'avenir de leurs enfants, le besoin de sauvegarder leur liberté, leur travail, leur temps. On peut même penser qu'ils se cachent à eux-mêmes un grand nombre de leurs mobiles. La raison de cette inconscience se trouve dans le caractère réactionnel, émotionnel et passager de certains comportements éducatifs : le but poursuivi ne se formule pas, si ce n'est au cours de conversations avec des personnes étrangères à la famille, ou à la suite de la constatation des carences éducatives. On peut dire que plus la famille vit repliée sur elle-même, et plus il y a de chances pour que l'éducation se poursuive de façon subconsciente. Si l'on constate des insuffisances dans l'équilibre de la personnalité de l'enfant, il n'est pas rare qu'on s'en prenne au tempérament ou à des influences extra- 
familiales. Ce sera pour nous un problème de découvrir dans quelles circonstances les parents en viennent à formuler une pédagogie consciente, qu'ils feront effort pour appliquer. Nous verrons le rôle d'institutions sociales diverses : de la tradition, du niveau d'instruction des parents, de l'école, des fréquentations sociales qui font sortir les parents de leur milieu traditionnel.

La pratique éducative apparaît comme un compromis entre la coutume, les conceptions régnantes dans la classe sociale, les théories pédagogiques et philosophiques, et une expérience quotidienne qui amène les parents "à en prendre et à en laisser". La part de ces facteurs est bien différente selon les familles, et la personnalisation de l'enfant s'en trouve orientée de façon parfois opposée.

\section{L'ÉDUCATION EN MILIEU RURAL ${ }^{1}$}

\section{Les familles observées.}

$1^{\circ}$ AUDE. - S'-J. - de - B. (à l'ouest du département). Les familles observées vivent dans la Piège ; région assez pauvre de polyculture vivrière : céréales, fourrage, quelques bovins ; élevage de volailles. Habitat très dispersé, petite propriété familiale $(25 \mathrm{Ha})$, peu de métayers et fermiers, peu ou pas d'ouvriers agricoles.

Famille 1 : P. 46, M. 44, G. 22, G. 14, G. $10^{2}$

Fermiers - tracteur - la radio -1 hebdomadaire agricole. Pas de loisirs, on ne quitte qu'exceptionnellement la ferme.

G. 10 est né avant terme (7 mois), après le décès tragique de l'un de ses frères. Les parents ont reporté sur lui tout leur attachement.

Famille II : P. 35, M. 40, G. 18, G. 8, F. 4.

Propriétaires aisés (650.000 fr. ?). Tracteur -6 bovins, 1 jument - radio -1 quotidien en période d'hiver -1 hebdomadaire agricole. Pas de loisirs, on ne quitte qu'exceptionnellement la ferme. Le père est un homme intelligent (éducation primaire). C'est le seul qui dans le village ait tenté de rationaliser son exploitation.

Famille III : P. 60 - M. $59-$ F. 11 + Jeune ménage : P. 35, M. 28, G. 3.

Petits propriétaires (400.000 fr.) tracteur -4 bovins - radio -1 quotidien -1 hebdomadaire agricole - famille nombreuse devenue récemment propriétaire. Pas de loisirs.

$F .11$ est la benjamine d'une famille nombreuse ( 9 enfants) tous les autres sont mariés - grande différence d'âge avec les autres enfants : l'avant-dernier a 12 ans de plus qu'elle. C'est la benjamine gâtée.

Famille IV : P. 39, M. 32, F. 11, F. 10.

Petit entrepreneur en maçonnerie sans ouvrier. Non imposé - Revenu 400.000 fr.

La fille aînée poursuit ses études dans un collège moderne. Pas de journal - pas de radio - 1 camionnette pour le travail.

Peu de loisirs.

\footnotetext{
1 . Nos remerciements vont ici à tous ceux qui ont bien voulu collaborer à notre enquête, et notamment à Mmes Scudier, Haure, Riou, institutrices, à M. Costes, qui ont bien voulu constituer quelques une des monographies que nous avons utilisées, et à $\mathrm{M}$. Bovard, qui nous a confié des rédactions où se reflète de façon très vivante la vie de ses élèves.

${ }^{2}$ Nous indiquons ainsi les âges du père, de la mère, des garçons et des filles, et le cas échéant la présence au foyer des grands parents. Nous mettons en italique l'âge de l'enfant observé.
} 
$2^{\circ}$ AVEYBON. - Les renseignements proviennent de régions diverses, mais toutes pauvres : Causse du Larzac d'une part : pays d'élevage de brebis, villages éloignés les uns des autres, religion catholique influente. Rouergue d'autre part : pays de polyculture, moins pauvre (on rencontre des propriétés assez grandes), où les fermes sont dispersées, l'influence du catholicisme assez grande. L'habitat est inconfortable, mais assez grand : une cuisine-salle commune, et des chambres où logent en général deux personnes.

Famille V(Larzac) : P. 37, M. 36, G. 8, F. 6, F. 1;6, A. G. M., G. P.

Revenu : 350.000 fr. (?) (n'est pas imposé). Une automobile, la radio.

Le père a le certificat d'études. On lit beaucoup (journaux, romans policiers).

La pêche, la chasse sont les distractions du père. On ne quitte jamais la ferme.

La mère jouit d'une grande autorité (quelques conflits entre les parents et le grand-père).

Famille VI (Rouergue) : P. 40, M. 40, G. 12, G. 10, G. 4, F. 0,6, G. M.

Revenu 300.000 fr. (?). Le père lit le quotidien et les publications agricoles.

On ne fréquente que les proches parents.

Famille VII (Rouergue) : P. 45, M. 38, F. 13, G. 12, G. 11, G. M. et G. P.

Revenu : 25 q de blé -25 hl de vin déclarés (la propriété a 7 ha).

Le père est aussi charron-charpentier. Les deux aînés, dans des collèges privés, ont obtenu une bourse entière.

P. et M. ont le certificat d'études.

La mère lit des romans. On reçoit des hebdomadaires catholiques.

Famille VIII (Rouergue) : P. 46, M. 46, G. 17, G. 14, F. 10.

Famille aisée (16 ha), qui modernise l'outillage.

On lit le quotidien régional, mais il n'y a pratiquement aucun loisir (acharnement au travail).

On ne fréquente que les parents.

Famille IX (limitrophe du Cantal) : P. 40, M. 37, G. 17, G. 14.

Famille aisée ; ferme moyenne, bâtiments plus grands que dans les autres fermes.

Confort (eau chaude - douche - four à gaz) Automobile - Tracteur - Un domestique - Famille assez exceptionnelle, qui fait figure de chef de file, et a de nombreuses relations dans le village.

Famille X (Causses de Séverac) : P. 32, M. 28, G. 11, G. 7, G. 4, G. P., frère du G. P.

Revenu : 1 million (?) - Conditions de vie archaïques - Pays pauvre. Le père a le $C$. E. P. Pas de loisirs culturels, peu de lectures, pas de relations.

Famille XI (Causses de Séverac) : P. 30, M. 29, G. 11, F. 10, G. 8, G. 2.

Revenu : 800.000 fr. (?) L'exploitation agricole est assez moderne.

Instruction du niveau du C. E. P.

Famille XII (Gros bourg) : P. 34, M. 30, F. 12, F. 9, G. M. et G. P.

Le père est artisan maçon, la mère ne travaille pas hors du foyer - La famille paraît aisée et a une maison confortable.

Le père a le brevet élémentaire, la mère le C. E. P. Les loisirs sont consacrés à la lecture, au jardinage, le dimanche à des promenades et au cinéma.

Le type de l'éducation dépend d'un certain nombre de facteurs que l'on pourrait appeler accidentels - si on les confronte aux facteurs sociologiques, auxquels ils sont pourtant inextricablement mêlés. C'est par exemple l'influence du type constitutionnel de l'enfant, plus ou moins bien accepté par la famille. C'est ainsi que 
G. I. $11^{3}$ est un enfant nerveux, impulsif, à la fois très affectueux et inhibé : venu un an après la mort accidentelle d'un frère âgé de 12 ans, il est traité avec indulgence par la famille, par le père principalement. Le rang de l'enfant joue un rôle considérable à la campagne : si l'aîné paraît présenter des qualités susceptibles d'en faire le successeur du père à la tête de la ferme (trop petite pour être partagée), le puîné sera souvent orienté vers des carrières de fonctionnaire ou d'artisan. C'est ainsi que G. II. 8 est le benjamin choyé : le père a formé en la personne de l'aîné un bon cultivateur, et ne se préoccupe pas de diriger son fils cadet, qui a la réputation d'un enfant entêté et "volontaire", et qui tient à garder cette réputation. Le problème se complique s'il s'agit d'une fillette : F. III. 8 est la benjamine gâtée ; anorexique, émotive, affectueuse, elle capte l'attention des parents et se satisfait de sa médiocrité, car les parents n'ont pas d'ambition pour une fille. De même faut-il prendre en considération les réactions des parents aux succès de l'enfant, comme à tout autre événement important de sa vie (accidents, maladie, réactions affectives aux frères et sœurs, aux parents et aux grands parents...). Ainsi F. IV. 11 est l'aînée qui réussit en classe, les parents la stimulent, sont exigeants envers elle - ce qui l'entraîne parfois à mentir - tandis qu'ils se désintéressent du travail de sa sœur P. IV. 10 qu'ils considèrent incapable de succès scolaire...

Sans faire abstraction de telles réactions parentales, nous aurons à observer surtout ce qui dans le climat éducatif provient essentiellement du milieu social, c'està-dire ce qui se présente avec une fréquence suffisante pour qu'on puisse y voir l'influence, non d'un trait familial, mais d'un trait social. Ce qui, également, est sanctionné par l'opinion publique du village. Et nous verrons que les sources de ces comportements sont à chercher, pour une part, dans les conditions de travail et, pour une autre part, dans la persistance de traditions et de croyances harmonisées à ces conditions.

La différence est grande entre les cinq enfants de St. J. que nous avons observés et que nous pourrions sommairement caractériser,

- G. I. 11 comme un nerveux, hyperémotif, qui fixe difficilement son attention, malgré une intelligence et une mémoire bonnes, arrêté dans son développement affectif ;

- G. II. 8 comme un enfant plein de vivacité, capable de fixer solidement des habitudes, intelligent, mais livré à lui même et porté à l'opposition aux parents ;

- $\quad$ F. III. 8 comme une fillette inhibée, non sevrée affectivement, émotive ;

- F. IV. 11 comme une fillette équilibrée sur le plan affectif et moteur, mais inhibée par la crainte des sanctions, et inégale du point de vue de l'attention ;

- F. IV. 10, peu émotive, mais peu capable de contrôle émotionnel, lente dans l'acquisition des habitudes comme dans la compréhension, comme une fillette amorphe et peu douée, résignée aux échecs. -

Quelles que soient ces différences, on découvre sans peine l'existence d'un type paysan : le langage apparaît assez tardivement ; les intérêts vont, chez les garçons, aux jeux violents et aux courses à travers champs, chez les filles, à des jeux plus calmes et ménagers ; la liberté d'action et d'allure est assez grande (quelque peu limitée par l'aide apportée aux parents) ; malgré les efforts du maître, l'horizon culturel reste limité : autant de traits que l'on peut rapporter à l'influence de la société.

Sur quelques points, 1a comparaison entre petits paysans de régions différentes fait bien ressortir cette influence de la société. C'est ainsi que l'importance du travail manuel des enfants, notée par de nombreux auteurs ${ }^{4}$, est variable dans les familles observées : elle

\footnotetext{
${ }^{3}$ G. ou F. désigne le sexe ; I, II, le numéro de la famille ; 5, 10, l'âge de l'enfant.

${ }^{4}$ Cf. Th. MAURETTE, H. GRATIOT-ÂLPHANDÊRY, P. Rossi etc. Loisirs et formation culturelle de l'enfant rural, P. U. F. 1956, p. 68 sq.
} 
est grande partout où il y a des troupeaux à garder (notamment dans la famille V), plus réduite à St J. ou dans le nord de l'Aveyron. Ou encore on constate que la déférence de l'enfant envers l'adulte n'existe pas à St J. : il tutoie tous les adultes et les appelle par leur prénom, comme le font les parents ; les jurons ne manquent pas dans ses propos; il en va autrement dans les villages de l'Aveyron...

\section{Les premières années.}

La fonction des soins donnés à l'enfant pendant les premières années est de permettre, avec la maîtrise du corps propre et son ajustement au milieu physique, la conquête d'un être social, constitué par les propriétés accordées à l'enfant et par les relations différenciées qu'il établit avec ses proches.

On constate assez souvent à la campagne la méconnaissance de l'importance de cette première étape de l'éducation. À la question concernant son rôle dans le développement de l'enfant, les mères répondent le plus souvent qu'elle doit lui assurer une bonne santé, et que cela seul importe Elles n'ignorent pas les principes élémentaires de pédiatrie concernant la régularité des repas et des périodes de veille et de sommeil, mais elles sont esclaves des travaux ménagers et, surtout, des soins à donner aux bêtes. Bien souvent, par ailleurs, les grand'mères conseillent l'observance des anciennes coutumes :

- "Il faut donner à téter dès que l'enfant pleure", ou, au contraire : "on doit le laisser pleurer tant qu'il n'est pas malade".

- "L'urine fortifie le bébé, il ne faut pas le changer trop souvent" -

- "II ne faut jamais laver le bébé, mais le laver avec de l'eau de cologne".

- $\quad$ "Pour qu'il ne prenne pas froid, l'emmailloter le plus longtemps possible".

La jeune maman accepte d'autant plus facilement la coutume que, si elle la heurtait, elle risquerait d'être critiquée, non seulement dans sa famille, mais encore par l'opinion du village.

Le rythme repas-sommeil-jeux étant irrégulier, l'entant devient tyrannique, y compris la nuit où il attend pour se rendormir quelques gorgées de lait ou quelques bercements. On lui donne fréquemment une sucette, qu'il conserve parfois au-delà de 2 ans (St J.). La mère n'a pas non plus le temps de s'occuper du dressage à la propreté : elle exigera la propreté à partir de 2 ans (VIII), à partir de 3 ans (IV); dans cette famille, les deux enfants n'ont pas porté de culottes avant 2 ans et demi ; et, laissés seuls pendant que les parents étaient aux champs, ils salissaient le plancher. Les jeunes parents cependant ont tendance à rompre avec la tradition.

Les carences sur le plan sensori-moteur et linguistique sont encore plus graves. Les mères, interrogées sur l'opportunité de donner des jouets , à l'enfant de moins de 2 ans, de lui parler, répondent que ce n'est pas nécessaire. L'enfant "ne comprend pas", jusqu'à un an tout au moins : c'est à partir d'un an qu'il devient intéressant (V), à cause de son sourire (VIII), de sa "roublardise" (V). Les parents lui parlent peu, et conservent, avec lui le langage "bébé" ; aussi restera-t-il longtemps au stade du langage puéril. À St J., les enfants de 4 à 5 ans qui entrent à l'école ont souvent une voix peu distincte, surtout ceux qui ont des frères aînés, et que les parents ont un peu délaissés. L'enfant reste seul dans son lit de longues heures (observation identique pour V et VIII), on le comprend à l'aide de quelques motsclefs qu'il accompagne de gestes imitatifs. 
Les jouets sont presque inexistants. Ce n'est guère qu'à Noël qu'on en achète. Les garçons ont des tracteurs (parfois donnés par des firmes), les fillettes ont des poupées. Ils en construisent avec des cercles, des vieilles bicyclettes, des boîtes. Parfois les parents, pendant l'hiver, leur fabriquent des "remorques". Les jeux sensori-moteurs sont, surtout chez les garçons, plus répandus que les jeux de fiction. Mais les enfants qui vivent dans les fermes isolées sont encore plus mal partagés.

L'éveil social est assez lent. D'une part, la vie familiale n'est pas variée et le travail absorbe les parents, d'autre part, l'enfant n'a pas la possibilité d'enrichir son expérience par des contacts extrafamiliaux, il baigne constamment dans la même atmosphère, si bien qu'il éprouve en face des étrangers un sentiment mêlé de crainte et de curiosité. C'est là peut-être le germe de cette attitude de prudence, de méfiance même, qu'on a souvent signalée chez les paysans. Il manque à l'enfant rural la multiplicité d'expériences qui lui permettrait de faire des comparaisons et de se placer à des points de vue divers.

F. V. 1;6 : elle est laissée au lit, éveillée, pendant un temps très long, car elle ne pleure pas : on est surtout content de la voir "sage". On s'intéresse aux quelques tours dont elle est capable, on essaye de deviner ses intentions : "On le voit aux yeux ce qu'elle veut dire", mais on a peur de "la gâter, et on la laisse pleurer si elle n'est pas malade".

Dans la famille VIII, la mère estime qu'il faut laisser les enfants pleurer si l'on pense qu'il ne leur manque rien et qu'ils ne sont pas malades. On doit leur infliger de légères punitions (tapes, privations de gourmandises) après deux ans. On doit les rendre sociables, les emmener dans de petites sorties chez les voisins.

Les travaux de la terre constituent le cadre essentiel de cette première pédagogie. On peut la caractériser comme assez libérale, non point par principe, mais parce que les parents manquent de temps. L'être est une force naturelle livrée à elle-même. Il est innocent : il brise tout, mais c'est un peu normal ; il est désordonné ; "on ne peut demander à l'enfant (de 2 à 6 ans) de ranger lui même ses affaires, il ne sait pas. II s'habillera seul à partir de 5 ans seulement. (Famille V). "II ne faut pas céder à ses caprices, mais on cède parfois pour avoir la paix". (VIII). Ainsi l'hétéronomie, en cette période, semble réduite. Les courses aux abords de la maison, les jeux avec les animaux, auxquels l'enfant s'attache vivement, créent une atmosphère joyeuse. Sur le plan de la nourriture cependant, il semble que l'on soit souvent assez strict : l'enfant "doit manger de tout" (VIII), "il ne sait pas s'il a assez mangé" (V).

$\mathrm{Au}$ total, cette éducation apparaît comme favorable au développement des fonctions motrices ; le petit rural est en bonne santé, hardi à l'égard des bêtes. Mais le langage est souvent en retard, et la vie imaginative surtout est réduite : les parents en effet ne portent aucun intérêt aux jeux de fiction et ne les encouragent pas en donnant des jouets II doit en résulter un certain retard sur le plan intellectuel, s'il est vrai que l'imagination ouvre la voie à la confrontation des possibles et du réel et favorise, indirectement, le développement de l'esprit critique. Par contre l'enfant trouve dans le cadre familial, à l'âge préscolaire, le climat affectif qui lui confère un sentiment de sécurité. On doit souligner ici le rôle des grands parents, dont l'indulgence bien connue est signalé par presque tous les enquêteurs ; c'est eux, aussi, qui ont le plus de temps pour s'occuper des enfants, et qui les gardent pendant que les parents sont aux champs. Leur pédagogie, si elle est 
moins rigide que celle des parents, est aussi moins stimulante, et ne semble pas susceptible d'enrichir beaucoup les activités des enfants.

\section{L'éducation de l'écolier.}

La période scolaire introduit des changements profonds, parce qu'il permet l'insertion de l'enfant dans un temps de progrès dont il n'avait guère la notion, parce qu'elle constitue le personnage de l'écolier, à qui doit être reconnu, par la famille elle-même, une certaine autonomie parce qu'elle permet à l'enfant de constituer avec ses pairs, sous la direction du maître comme dans les jeux indépendants, une série de relations sociales complexes. Les parents vont rapidement se trouver devant un être nouveau, et les lacunes de leur pédagogie proviennent pour une grande du fait qu'ils comprennent mal cette mue, et ont tendance à attirer l'enfant à eux.

Les transformations introduites par la vie scolaire ne sont peut-être pas aussi importantes que celles qui se produisent en ville. L'enfant en effet connaissait déjà les petits camarades qu'il retrouve à cinq a son entrée à l'école, et comme ils sont peu nombreux, ils forment famille élargie. Ils sont façonnés par un maître qui les connaît bien qui les connaît au-delà de la classe ; ils jouent aux mêmes jeux ; ils écoutent les mêmes paroles : l'école ne procure pas les contacts diversifiants qu'elle peut procurer à la ville. À population homogène, population scolaire homogène : vivant de façon identique, ils ne peuvent s'enrichir mutuellement et découvrir la complexité de la société et des problèmes qu'elle pose. Qualitativement cependant, l'école crée une rupture, en introduisant des attitudes peu fréquentes dans les familles.

En premier lieu, elle donne à l'enfant un personnage : il doit acquérir le sens de l'ordre, de la discipline individuelle et collective, de l'effort suivi. (À St J. tout devoir mal fait doit être refait à la maison ; la dictée qui comporte trop de fautes y sera recopiée, ainsi que les règles non sues). L'école rend l'enfant plus obéissant, et en même temps moins timide. Elle révèle, aussi, des intérêts que la famille ne peut pas toujours développer : sur le plan intellectuel bien entendu, mais aussi sur le plan moral : sentiment de solidarité, de générosité, attachement à la vérité scientifique, à l'esthétique, à l'hygiène même... Un exemple de ces apports moraux : l'école, étant mixte, met la fille sur un plan d'égalité avec le garçon : attitude tout opposée à celle de la société familiale, où la jeune fille et la femme ne sont pas considérées comme les égales du jeune homme et du mari. Le personnage de l'écolier a deux dimensions bien distinctes, selon qu'il se situe par rapport aux camarades de sa division ou par rapport à ceux des autres divisions. À ce dernier point de vue, il est celui qui progresse : il se rapproche du certificat, ses connaissances s'accroissent, il peut, le cas échéant, servir de moniteur auprès des petits. Comparé à ses pairs, il est "bon" ou "mauvais" : les différences sont considérables, et l'enfant, même si les compositions et les notations systématiques sont éliminées par le maître (cas de St J.), prend vite conscience de sa valeur et de ses insuffisances.

En dernier lieu, l'école révèle à l'enfant le monde culturel et les activités de création intellectuelle. Il y acquiert le sens du travail minutieux, il apprend à faire preuve d'initiative et d'inventivité. Il s'adonne également à des jeux collectifs qui, 
par leurs règles, leurs obligations, les satisfactions personnelles qu'ils apportent contrastent fortement avec les jeux presque toujours solitaires de l'enfant dans sa ferme.

Nous avons un bon exemple de cet apport de l'école à St J. : le maître a créé, en 1952, une coopérative scolaire, qui s'est d'abord donné pour tâche d'organiser un voyage à la mer, puis de donner des séances de cinéma (inconnu jusqu'à ce jour de la plupart des enfants et des femmes, il a un très vif succès chez les adultes, qui éprouvent la joie de se retrouver en société.). Mais la fête de Noël, où les enfants donnent un spectacle, a un succès plus grand encore, en révélant aux parents tout ce que peuvent faire les enfants. - L'école possède en général une bibliothèque, dont les livres et les hebdomadaires sont lus, non seulement par les enfants, mais par les parents (dans tous les villages où l'enquête a été effectuée).

Comment les parents réagissent-ils à ce petit écolier, dont ils sentent bien qu'il leur échappe quelque peu, et qu'il devient souvent plus cultivé qu'eux-mêmes ? Ils passent par des réactions ambivalentes. D'une part, ils désirent pour leurs enfants la meilleure réussite scolaire, d'autre part ils sont tentés bien souvent de ne pas considérer le travail scolaire comme un véritable travail, et ils exigent de leurs enfants une aide importante dans les activités de la ferme.

L'entant ne peut pas travailler dans une pièce tranquille (dans toutes les familles). Les parents ou les aînés s'occupent de son travail irrégulièrement (I. V. IX. X. XI) ou pas du tout (II.. III) ; font cependant exception les familles IV. VI. VII. VIII. XII, mais \}a surveillance n'est jamais très grande. C'est en général le succès spontané de l'.enfant qui amène les parents à le "pousser" (IV. VII).C'est aussi l'amour-propre ; à la question : "quelles satisfactions vous procurent les enfants?", la réponse la plus fréquente est relative aux bons résultats scolaires ; en deuxième lieu vient la satisfaction d'être aidés par les grands.

Le petit rural se trouve bien peu -encouragé au travail intellectuel par l'ambiance familiale. On lit peu, en général.

À St J., le facteur distribue en moyenne, pour 70 familles, une trentaine de journaux et une dizaine de lettres familiales. Sur ces 80 journaux, une vingtaine de quotidiens seulement ; certains ne s'abonnent que pour la période d'hiver. Il n'y a pas de bibliothèque communale dans la région. Dans l'Aveyron, l'importance de la lecture semble plus grande (rôle de la presse catholique).

\begin{tabular}{|l|l|l|l|l|}
\hline Famille & Quotidien & $\begin{array}{l}\text { Hebdomadaire } \\
\text { local }\end{array}$ & $\begin{array}{l}\text { Hebdomadaire } \\
\text { agricole }\end{array}$ & Romans \\
\hline I & & & + & \\
\hline II & + (hiver) & & + & \\
\hline III & + & & + & \\
\hline IV & & & & \\
\hline V & + & + & & + \\
\hline VI & + & & + & \\
\hline VII & & + & + & + \\
\hline VIII & + & & & \\
\hline IX & & + & & \\
\hline X, XI, XII & + & & & \\
\hline
\end{tabular}

II existe une coupure nette entre l'atmosphère de la classe et celle de la famille. Pour peu que l'enfant ne réussisse pas en classe, la décision est prise : il sera un paysan et c'est aux travaux des champs qu'on va l'entraîner, afin qu'il y prenne goût. On découragera les jeux et les lectures qui pourraient l'en détourner. 
Les enquêteurs sont unanimes sur ce point : en général c'est au fils aîné qu'est dévolue la fonction de remplaçant du père ; il est mis au courant de cette «vocation », et en vient rapidement à vouloir ressembler à son père (Famille I. II. III. V. VII. IX. X). Il arrive qu'à l'invitation de l'instituteur, le fils aîné soit orienté vers la continuation des études : les parents pourtant l'acceptent mieux pour un puîné. (Les familles des propriétaires exploitants comptent en moyenne deux enfants dans la Piège ; dans l'Aveyron, les familles observées comptent en moyenne 3 enfants. C'est chez les paysans sans terre (métayers) que l'on trouve des familles de plus de 3 enfants).

Les jeux sont mal tolérés. Dans l'Aude comme dans l'Aveyron, ils sont considérés comme un luxe, qu'il ne faut pas encourager car il pourrait aboutir à rendre l'enfant paresseux. Les parents n'aiment pas voir les enfants jouer pendant qu'euxmêmes travaillent, et ceux qui le tolèrent sont en général mal considérés par l'opinion publique. Les fillettes, cependant, semblent plus libres que les garçons, auxquels est confiée la garde du bétail, à partir de 8 à 9 ans.

G. VI. 10. Les parents considèrent le jeu avec les camarades, non comme une chose importante pour le développement de l'enfant, mais en quelque sorte comme un mal inévitable : ils préféreraient que l'entant soit spontanément "sérieux".

G. VIII. 14. Laisser jouer les enfants, c'est une récompense qu'on leur accorde une fois le travail fait. On les laisse alors se promener à bicyclette. À la question : "doit-on donner des livres ou des illustrés ?", les parents répondent : "ils n'en demandent pas".

À St J. dès 7 à 8 ans les garçons se voient confier la garde du troupeau (vaches et bœufs), dès la sortie dès la sortie de classe parfois, et dans tous les cas les jours de congé. Ce n'est guère qu'après 12 ans cependant que commence l'entraînement au travail agricole véritable : après la classe, le garçon renouvelle la litière, il participe à la fenaison, à la moisson, aux labours... Il n'est pas récompensé pour ce travail, il l'effectue parce qu'il doit l'effectuer. II se plie d'autant plus volontiers aux exigences de ses parents qu'il est fier de les aider, et qu'il prend conscience de sa force.

V. G. 8. et sa sœur F. 6 gardent les porcs matin et soir.

VI. G. 10. participe peu ou prou à tous les travaux de la ferme. Son père l'initie à la mécanique.

L'imitation du père est notée par tous les observateurs comme jouant un rôle décisif dans cet entraînement aux travaux.

IX. G. 14. fournit un gros travail : levé tous les jours à 6 h 30, il travaille le soir en rentrant de classe, et en été il va garder les bêtes. "C'est ce que font au minimum tous les enfants de ce milieu. On leur demande en outre de petits services : casser le menu bois, porter le fourrage aux bêtes, le grain aux poules. G. s'intéresse beaucoup aux travaux de la ferme ».

\section{L'éducation morale.}

Travail scolaire peu encouragé, jeux considérés comme des activités accessoires, sinon condamnables, participation aux tâches agricoles, à ces trois points de vue, on peut dire que les parents méconnaissent quelque peu l'originalité de la période enfantine. Cela se reflète dans une conception généralement sévère de l'éducation de la personnalité. Les punitions, souvent corporelles, y jouent un grand rôle, beaucoup plus grand que les récompenses.

Cette sévérité n'est cependant pas l'expression d'un système éducatif : pour qu'il y en ait un, il faudrait que les parents aient davantage de temps, qu'ils puissent s'imposer une discipline, une maitrise de soi, des renoncements, autant de contraintes que le travail de la terre leur interdit d'exercer sur eux-mêmes. Il s'agit d'une sévérité discontinue, et comme naturelle, qui dans certains cas peut céder la place à l'indulgence ou à l'indifférence. 
G. I. 10. Enfant nerveux, aux réactions motrices brutales et désordonnées, émotif et instable. Parce qu'il est le dernier-né, qu'il est affectueux et qu'il a coûté beaucoup d'efforts dans les premières années, le père lui pardonne tout. Il est très difficile à diriger. Seule la mère punit.

G. II. 8. Enfant intelligent, mais livré à lui-même, il est puni de façon très irrégulière en fonction de l'exaspération des parents. Ils lui ont donné une réputation d'entêtement dont il est fier.

F. III. Benjamine d'une famille de 7 enfants, elle est généralement choyée et on satisfait ses caprices ; d'où une absence d'effort et d'intérêt.

F. IV. 11. Le père est maçon (petit entrepreneur). La mère veut faire de sa fille une fonctionnaire et se montre sévère, notamment sur le plan des mensonges.

F. IV. 10. Moins douée que sa sœur, inerte; ses parents la persuadent de son infériorité et ont tendance à ne pas lui demander d'effort.

P. V. 6. Le père réprimande ; il est persuadé qu'il ne faut pas céder aux caprices de l'enfant. La mère est d'accord avec lui, et la trouve jalouse et capricieuse. Ils sont préoccupés par les disputes entre elle et son frère aîné, et leur sévérité se heurte à l'indulgence des grands parents.

G. V. 8. Le père est très strict sur l'ordre "la première qualité d'un homme "), il gifle parfois : "il faut les corriger mais être juste". Père et mère assimilent éduquer et obtenir l'obéissance. De cet enfant qui doit leur succéder à la ferme, ils attendent avant tout qu'il prenne le goût du travail et ils veillent à le rendre honnête.

G. VI. 10. Le père est très autoritaire, il est craint par l'enfant, qui n'ose guère désobéir et exprimer ses désirs. Les châtiments corporels sont parfois utilisés. L'enfant est peu soucieux de prendre des initiatives. La maîtresse déclare : "Le père a modelé avec succès une personnalité plastique et sans relief, il a développé le sens pratique et l'esprit logique."

G. VII. 11. La mère seule est sévère. Le père est bienveillant (c'est le benjamin). L'enfant est équilibré.

G. VIII. 14 et P. VIII. 10. Le père seul est sévère ; le garçon l'imite et veut devenir cultivateur, il est très intéressé par la modernisation de la ferme.

G. IX. 14. Les parents sont partisans d'une éducation sévère mais confiants. Le fils est obéissant ; il admire ses parents et ne leur pose pas de problèmes.

Ainsi, les conceptions relatives à la sévérité sont des plus diverses : un fond de rudesse, qui découle de la vie harcelante du paysan, de son sens du devoirtravailler, n'empêche pas les parents de se montrer indulgents, et même faibles, par exemple pour le dernier-né. Les rivalités entre frères sont assez fréquentes et préoccupent les parents; à la question : "comment éviter la jalousie entre frères et scurs ?", on trouve la réponse : "donner la même chose à tous, mais c'est difficile..." (V). Ces disputes provoquent maintes fois des petits drames familiaux.

L'éducation apparaît, en fait, conflictuelle, libérale et cependant rétrécissante. Il ne fait aucun doute qu'elle laisse beaucoup de libertés aux enfants et, sous bien des aspects, on pourrait la dire marquée d'un naturalisme subconscient, car elle n'a pas le souci d'inculquer méthodiquement des habitudes techniques, des éléments de civilité, des principes cohérents de morale. Il n'y a pas de surveillance précise, constante, des rythmes de la vie biologique, des jeux, des travaux scolaires. Les talents et les carences sont mis au compte de l'hérédité et du tempérament : ils ne doivent rien à l'éducation. Nous retrouverons des habitudes et des croyances analogues à la ville, mais elles sont loin d'y être aussi répandues et aussi tyranniques qu'à la campagne.

Cette liberté primitive favorise un certain goût de l'aventure, les escapades dans les champs, les jeux de compétition : courses, luttes, batailles, les activités de 
construction utilitaire dans l'atelier du père. Elle entraîne naturellement bien des conflits avec les parents, qui laissent faire mais ne sont pas compréhensifs.

Quelques extraits de rédactions libres (Commune de Condezaygues, dans le Lot et Garonne) nous donnent une idée de cette vie de liberté.

B. B. (G. 10) : "Ce jour-là, je ne savais que faire. Je me dis : Si j'allais à la poursuite des pigeons avec la chienne? ... Naturellement la mère le réprimande ; elle m'a donné une bonne correction et je suis allé au lit."

B. C. (G. 9) joue aux Orsala (équilibristes) dans une prairie.

C. F. (F. 11.6) joue avec son frère sur un scooter en planche "qui a eu de cruelles opérations".

J. (G. 9) fabrique une cage avec une caisse.

L. J. (F. 11) et sa sœur jumelle : "Paulette installe une couverture pour jouer à la lutte. La poussière s'élève, les tourterelles applaudissent par des roucoulements sans fin. Je gagne la partie. Paulette se met à pleurer. Maman arrive et nous frappe. Je dis : Paulette, nous recommençons?... Je saute sur Paulette, deux coups de poings, aïe, aïe, aïe !"

II résulte de ce mode de vie une certaine maîtrise de soi, que vient accroître, chez les garçons, l'imitation du père. Car à partir de 12 à 13 ans, il est fier d'exécuter les travaux des hommes. À la sortie de l'école, il entre dans la catégorie des producteurs, et ce n'est pas sans une certaine gravité qu'il remplit ses nouvelles fonctions. Il passe par une période de refus de ce qui était l'enfance : à St J. par exemple, il lui arrive d'affecter une pointe de dédain à l'égard des femmes, et même de sa mère. Il abandonne le français pour la langue d'oc, il s'écarte de tout ce qui a trait à l'enseignement. La crise d'originalité juvénile consiste essentiellement en une tentative pour s'éloigner des tutelles qui ont dominé son enfance.

On découvre vite, cependant, les limites de la personnalisation du petit rural. Elles tiennent à deux facteurs principaux : au manque d'autonomie, d'initiative et d'inventivité, et à l'insuffisance des contacts humains.

La liberté concédée à l'enfant ne l'est pas pour construire. Elle ne conduit pas à l'unité. Ou bien il est livré à lui-même dans des activités désordonnées, ou bien il est très strictement guidé. En dehors de la classe, où il lui est possible de créer, mais selon des normes évidemment préétablies, sa vie est essentiellement d'obéissance (exception faite pour quelques enfants tyranniques, que les parents ne savent pas diriger).

On peut mesurer cette hétéronomie tant d'après l'importance des travaux manuels et des punitions que d'après l'absence assez fréquente de jeux et de lectures formateurs.

Dans une rédaction, une fillette de 13;6 raconte que pour s'endormir, elle pense à son travail journalier: "J'ai tricoté, mais cela ne va pas. Demain je le démolirai. J'ai fait la lessive ; Maman m'a dit que c'était bien. J'ai gardé ma petite sæur; pendant que je tourne le dos, elle me fait toutes les bêtises possibles. Et ma mère m'administre toutes les corrections nécessaires. Enfin je suis allée chercher des pissenlits".

Dans une famille nombreuse, une fillette $(13 ; 6)$ évoque les coups de bâtons, les gifles reçues et les joues rouges. Son frère $(11 ; 6)$ raconte son angoisse dans le récit d'une promenade : dans une cabane "deux gros trous laissaient voir la lumière solaire. D'énormes arbres semblaient dévorer ce pauvre petit monceau de pierres et de tuiles. Une grande tristesse y régnait : un lézard se promenait, morne, sur la fenêtre délabrée... quand je revenais... les grands arbres paraissaient des hommes. Je ralentissais le pas parce que ma mère me disait que des hommes armés d'un grand sac prenaient les enfants. il me semblait sentir une grosse main qui me prenait la gorge". 
D'autres enfants réagissent aux mauvais traitements en concevant de l'hostilité pour l'un des parents. Dans une famille où le père est craint pour sa brutalité, les enfants sont opposants ; une fille $(13,6)$ raconte avec humour sa joie à voir son père pester après une bêche disparue ; et dans une autre, le fils $(11 ; 6)$ très émotif, évoque ses peurs dans le lit, la nuit, comment il a pris son père pour un bandit, et il conclut : "Aujourd'hui, j'ai reçu deux corrections, quelques gifles. J'ai fait une charrette de foin, qui d'ailleurs s'est démolie" ... L'attachement à une bête, à une poupée, est souvent très vif : il s'agit là de compensations, comme le sont aussi les jeux violents et les disputes entre camarades.

Le petit rural est en mesure d'exercer sur le milieu des entreprises multiples. Mais il n'est soutenu ni par les adultes, ni par le milieu culturel : par les lectures que pourrait faire l'enfant et les récits qu'il pourrait entendre, et qui pourraient l'amener soit à des fictions, soit à des œuvres. La valorisation s'effectue dans le travail : l'enfant rêve de pouvoir conduire le tracteur, ou bien d'avoir, en possession "personnelle", une bête domestique : un mouton, un veau, voire un cheval, dont il a à s'occuper, objet de ses soins, de son affection, et de sa fierté. Les visées, même à l'adolescence, restent courtes : le détour par le monde culturel que révèlent les livres ne peut pas s'effectuer.

C'est là la deuxième carence de la formation du petit paysan : il ne peut pas s'évader de son coin de terre, il se représente mal quelles sont les conditions de vie du citadin, quelles sont les mentalités des hommes des autres professions et des autres classes. Il est vite pris dans le complexe du paysan moderne : à la fois fier de son travail, dont personne ne veut plus, et désireux de sortir de sa condition : mais il ne se renseigne pas vraiment sur celle des autres.

Nous pouvons juger de l'ouverture sociale d'après la nature des loisirs et des relations extra familiales, la lecture, les intérêts culturels, le métier désiré. Dans les douze familles observées (10 cultivateurs, dont deux métayers, deux propriétaires aisés, huit petits propriétaires ; deux artisans maçons) les loisirs culturels se répartissent de la façon suivante (il s'agit des activités de la famille, sauf pour les points 7, 8 et 9).

\begin{tabular}{|c|c|c|c|c|c|}
\hline & $\begin{array}{l}\text { Métayers } \\
\text { Petits propr. } \\
\text { S'J }\end{array}$ & $\begin{array}{l}\text { Petits } \\
\text { propriét. } \\
\text { Aveyron }\end{array}$ & $\begin{array}{l}\text { Propriétaires } \\
\text { aisés }\end{array}$ & Artisans & $\begin{array}{l}\text { Total } \\
\text { (12 familles } \\
\text { et } 15 \text { enfants) }\end{array}$ \\
\hline 1 - Cinéma & $\begin{array}{l}3 \text { ciné post } \\
\text { scolaire }\end{array}$ & & 1 & 2 & 6 \\
\hline 2 - Fêtes locales & 3 & 5 & 2 & 2 & 12 \\
\hline $\begin{array}{l}3 \text { - Messe dominicale } \\
\text { Hommes } \\
\text { Femmes }\end{array}$ & $\begin{array}{l}0 \\
0\end{array}$ & 5 & $\begin{array}{l}2 \\
2\end{array}$ & & $\begin{array}{l}2 \\
7\end{array}$ \\
\hline $\begin{array}{l}4 \text { - Relations familiales } \\
\text { seulement } \\
4 \text { - Relations familiales et } \\
\text { extra familiales }\end{array}$ & $\begin{array}{l}3 \\
3 \text { (hiver) }\end{array}$ & 4 & $\begin{array}{l}1 \\
1\end{array}$ & 1 & $\begin{array}{l}9 \\
6\end{array}$ \\
\hline 5 - Quotidien. & 1 & 5 & 2 & 1 & 9 \\
\hline 6 - Intérêt pour la lecture. & 0 & 3 & 1 & 1 & 5 \\
\hline $\begin{array}{l}7 \text { - Livres de la Biblioth. } \\
\text { scolaire }\end{array}$ & 3 & & 1 & 1 & 5 \\
\hline $\begin{array}{l}8 \text { - Illustrés pour enfants } \\
\text { Livres d'enfants }\end{array}$ & $\begin{array}{l}1 \\
3\end{array}$ & $\begin{array}{l}3 \\
2\end{array}$ & $\begin{array}{l}1 \\
2\end{array}$ & $\begin{array}{l}1 \\
2\end{array}$ & $\begin{array}{l}5 \\
9\end{array}$ \\
\hline $\begin{array}{l}9 \text { - Vocations } \\
\text { agriculteur } \\
\text { mécanicien } \\
\text { instituteur } \\
\text { non précisée }\end{array}$ & $\begin{array}{l}1 \\
1 \\
1 \\
1\end{array}$ & $\begin{array}{l}1 \\
2 \\
3\end{array}$ & $\begin{array}{l}1 \\
1\end{array}$ & $\begin{array}{l}2 \\
1 \\
\end{array}$ & $\begin{array}{l}3 \\
4 \\
3 \\
5\end{array}$ \\
\hline
\end{tabular}


Bien qu'on ne puisse tirer de conclusion de chiffres aussi restreints, il est frappant de constater combien les familles paysannes se fréquentent peu : parmi ceux-là même qui reçoivent des membres de leur famille, il n'en est que 3 pour le faire de façon régulière. L'attrait pour la mécanique est considérable, en raison des autos et des tracteurs, et parce que c'est sous cet angle que le monde moderne apparait à l'enfant Les deux fillettes qui désirent devenir institutrices sont filles d'artisans : ce n'est sans doute pas pur hasard.

Même quand le journal, la radio, quelques livres, introduisent à campagne les opinions et les préoccupations du monde moderne, les enfants ne s'y intéressent pas vraiment, fût-ce aux approches de l'adolescence. Plus encore que ceux de la ville, ils vivent dans l'ignorance de ce qui n'est pas sous leurs yeux. Tout le reste, ce sont des réalités lointaines et un peu irréelles, dont le maître parle en classe, dont la radio les entretient, mais tout se passe comme si l'on n'y croyait pas tout à fait. Ce qui compte c'est l'ensemble des relations sociales, c'est l'obligation où est l'enfant de jouer, à la maison et à l'école, les divers rôles que le milieu proche lui assigne.

La pédagogie de la personnalité morale de l'enfant est ainsi profondément marquée par le primat du travail et par l'isolement. L'entraînement au travail, son acceptation facile par l'enfant donnent incontestablement au petit rural une sorte de robustesse morale. Mais il lui manque le désir d'une pensée et d'une action vraiment autonomes. L'école ne peut pas initier les enfants de moins de quatorze ans à la réflexion sur les problèmes modernes, à l'esprit critique, à la recherche de solutions nouvelles. Ils sont trop jeunes : seraient-ils plus âgés, ils viennent d'un milieu trop homogène pour que des discussions puissent s'instaurer utilement. Quant aux activités autonomes, elles sont rares à la campagne : les jeunes sont trop placés sous la double dépendance de leurs parents et de la tradition pour oser innover en-dehors du jeu.

L'imprégnation da l'entant par le milieu s'effectue sous deux formes : par l'imposition d'une discipline rigoureuse ( $80 \%$ des cas observés), mais aussi, dans les familles "libérales", sous l'angle de la discipline, par l'observance inconditionnée de rites auxquels l'enfant se plie tout naturellement. C'est ainsi que dans l'Aveyron on suit les offices religieux, on est soumis à l'influence de l'Eglise ; dans la Piège, seules les femmes suivent les offices et le clergé n'a pas d'influence. Mais dans les deux régions, il ne s'agit nullement d'actes délibérés : les problèmes religieux ne sont pas discutés, ils n'intéressent pas vraiment l'enfant ou l'adolescent.

Aux questions : "l'entant cherche-t-il à s'affranchir de son état de sujétion ? Peut-on saisir en lui un désir d'autonomie ?", les enquêteurs répondent en général (dans 10 cas) par la négative, tout en observant parfois des velléités soit de révolte, soit d'activités solitaires (dans 6 cas). Est-ce à dire que l'enfant ne soit pas sociable ? II n'en est rien ; il aime parler et jouer avec les petits camarades ; qu'il vive dans l'isolement de la ferme ne signifie pas qu'il soit "sauvage". Mais en fait, il ne s'ouvre pas à des réalités multiples, parce que les conditions économiques de la petite exploitation, qui attirent les gens à elle impérativement, ne le lui permettent pas.

Nous ne dirons pas qu'il n'y a pas de pédagogie morale : la lutte contre le mensonge, contre la paresse, contre l'égoïsme (notamment dans les rapports entre frères et sœurs) fait l'objet des soins permanents des parents comme du maître. Mais il y a du vrai dans cette remarque de l'enquêteur qui dit: "L'initiation aux questions morales est du ressort du Curé qui apprend le catéchisme et de l'instituteur qui essaie d'inculquer des notions de morale appliquée. Il arrive parfois que sous l'angle la propreté, de l'ordre, de la générosité... les exemples familiaux laissent 
un peu à désirer". Ce jugement ne serait cependant pas tout à fait juste si on méconnaissait la pression du travail et des traditions.

\section{Résumé et conclusions.}

1. - L'éducation à la campagne semble beaucoup plus homogène, d'une famille à l'autre, qu'elle ne l'est à la ville. Nous avons relevé de grandes différences sur un certain nombre de points importants ; sur le chapitre de la discipline, sur celui de la lecture, sur celui enfin des aspirations. Trois influences diversifiantes jouent ici un rôle important.

- La richesse, en premier lieu : il est évident que le petit métayer ne peut se procurer le poste de radio, ne peut s'abonner à des journaux comme le propriétaire aisé. C'est aussi en fonction de la richesse que s'effectuent les différenciations sar le point de savoir quel sera l'avenir de l'enfant.

- Il faut aussi tenir compte de l'importance de l'agglomération : autre sera l'éducation dans une ferme isolée et dans un village proche d'une petite ville : les traditions ont plus de chance de persister dans le premier cas. Une autre influence du milieu géographique et historique se traduit dans l'importance plus ou moins grande accordée par les parents aux disciplines d'inspiration religieuse : la différence est nette de ce point de vue entre la Piège et l'Aveyron; le prêtre y joue un rôle bien différent, et, par contre coup, l'école.

- Le troisième facteur de différenciation nous a paru se trouver dans le rang des enfants : nous avons relevé combien le benjamin était, dans bien des cas, traité avec plus d'indulgence que les aînés, chargés d'aider le père, et entraînés à devenir des hommes capables de le remplacer. Dans le même ordre d'idées, la différence est nette entre fillettes et garçons : on semble plus exigeant à l'égard de ces derniers, qui sont introduits plus jeunes dans le circuit du travail.

Mais à ces facteurs de diversification s'opposent et les exigences du travail, et le poids des traditions : il y a bien un type général d'éducation rurale, qui est sans doute l'une des sources de la personnalité paysanne.

2. - L'un des aspects fondamentaux de cette éducation réside dans la lenteur des processus de socialisation : le langage tardivement acquis, les jeux collectifs souvent restreints au groupe des frères et sœurs, et souvent concurrencés par le travail, les contacts culturels réduits à la lecture de quelques journaux, et à l'initiation scolaire, tout cela n'est guère propice à la formation d'une personnalité diversifiée, aux réactions sociales souples et riches.

La solitude est fréquente dans la vie du petit rural : tout jeune on le laisse seul dans la maison quand on se rend dans les champs ; il fait de longs trajets dans la campagne pour rejoindre l'école bien souvent sans compagnie ; en classe même, il se trouve avec des enfants plus âgés ou plus jeunes que lui, avec lesquels il n'ose pas jouer. Et il est seul pour garder les bêtes, traire les vaches ou les brebis, nettoyer les étables... Son caractère doit s'en trouver profondément marqué. 
3. - Ce qui frappe aussi, c'est un mélange original de liberté et d'hétéronomie. L'enfant n'est pas tant libre que livré à lui-même : du moins n'est-il pas sans cesse surveillé, et on ne trouve pas non plus chez les parents ces formes d'affection passionnée et captative qui paralysent l'enfant. Mais ce n'est, ni une pédagogie systématiquement libérale ni une pédagogie soucieuse de développer une personnalité créatrice.

Éduquer un enfant, c'est à la campagne savoir lui interdire tout ce que la morale traditionnelle considère comme mauvais, c'est, surtout lui donner le sens du devoir, du travail-devoir : nous sommes fort loin d'une éducation qui se confierait à la nature de l'enfant. C'est que cultivateur est conscient de la peine qui lui coûtent ses récoltes, il sait qu'il doit inculquer l'ardeur au travail à ses enfants. Nous avons vu comment il y parvient : en faisant de ceux-ci des associés, en leur donnant le sens de la propriété, en les rendant responsables d'un coin de jardin ou d'une bête. Naturellement, rien dans une telle éducation ne peut favoriser le goût de l'invention. (On note un léger changement dans 1es familles où le fils a suivi des cours d'agriculture).

La personnalisation s'accomplit essentiellement par l'acquisition des techniques que transmettent les parents, et non par l'élaboration de projets personnels. Pour que l'adolescence puisse concevoir le désir de créer du neuf, il faut qu'elle passe par des conflits : or, s'il peut se produire des conflits affectifs multiples dans les familles, ils ne vont pas jusqu'à provoquer des échanges d'idées, des discussions. L'adolescent vit sur le double legs du savoir scolaire et du savoir-faire professionnel, et il n'a guère l'occasion de discuter sur les questions religieuses, politiques, sociales.

L'enfant est happé par la vie familiale, il vit pour elle, en fonction d'elle et dans un milieu, homogène par sa composition sociale, amorphe dans sa structure (puisque les familles sont pratiquement indépendantes les unes des autres); il s'oriente vers cette forme d'individualisme paysan qui reflète essentiellement le type de la propriété et du travail que celle-ci a imposé.

4. - De quels problèmes pédagogiques les parents prennent-ils conscience ? Les divergences sur le problème de la sévérité entraînent des discussions, soit entre le père et la mère, soit entre ceux-ci et les grands parents. C'est surtout l'indulgence qui semble créer des problèmes ; l'enfant devient ingouvernable : "on ne sait plus quoi en faire", ou bien tombe malade si l'on essaye de prendre barre sur lui. L'indulgence est mal vue à la campagne, car elle est incompatible avec la formation d'un bon travailleur.

La réflexion pédagogique ne va cependant pas très loin : on se borne à constater les divergences, et à exercer une action compensatrice de celle du parti opposé. Une question qui revient fréquemment concerne les disputes entre frères et sœurs, que l'on a tendance à blâmer violemment, au lieu de prendre conscience de leur origine dans les comportements parentaux.

Le problème du choix du métier ne semble pas préoccuper beaucoup les parents : tantôt il est résolu automatiquement par l'accès à la propriété, tantôt on oriente l'enfant vers le métier d'un parent, tantôt on se fie à l'instituteur ou au prêtre pour le "pousser" jusqu'à un cours complémentaire ou un collège.

Quelques parents ont pris conscience que l'action éducative doit changer avec l'âge : "on le fait travailler davantage, nous surveillons nos conversations". Mais 
généralement les réactions à cet égard sont subconscientes. Les problèmes économiques sont exposés librement; mais les problèmes sociaux ou politiques ne sont abordés en famille que de façon assez laconique (on évoque les luttes du passé et on y reste fidèle). Les problèmes sexuels ne doivent pas être abordés (l'enfant apprendra de lui-même) : l'opinion est générale sur ce point. Les problèmes de l'éducation morale ne sont pas pensés de façon systématique, ou plutôt le système est inexprimé. II n'y a pas lieu de parler d'éducation esthétique, si ce n'est pour la mode, chez les jeunes filles.

La prise de conscience de certains problèmes pédagogiques s'opère par le canal des institutions fondamentales, et en fonction des transformations qui s'accomplissent en elles. Faut-il que l'enfant poursuive ses études après 14 ans ? C'est la crise de l'agriculture française qui oblige les parents à se le demander. Faut-il lui permettre de voyager, de partir en colonie de vacances ? Ce sont les initiatives de l'instituteur, fondateur d'une coopérative scolaire, animateur d'une chorale de jeunes, qui persuaderont les parents, et le maître apparaît ici comme le propagandiste d'un mouvement culturel caractéristique de notre époque, où le voyage, le cinéma ont une place prépondérante. Son rôle peut être décisif.

Il est des problèmes qui n'ont été posés aux parents qu'à la suite d'échecs subis dans l'éducation des premiers-nés ; la correction de leurs méthodes peut alors être excessive : il semble que ce soit le cas dans les familles I et II où les parents ont été conduits à une indulgence extrême, suivie de difficultés nouvelles. Dans ce cas, les critiques que leur adressent les parents ou des voisins peuvent contribuer à un changement d'orientation, mais nous avons vu que les relations entre familles sont assez rares.

L'influence des médecins celle des parents qui habitent la ville sont plus nettes ; il semble bien que la pédiatrie des grand'mères soit en voie de disparition dans les jeunes ménages. Le rôle de la presse et de la radio est aussi à considérer : véhicules des "idées de la ville" elles ne sont pas immédiatement écoutées ; il faut que l'ensemble ou la pression insistante des proches convainquent les parents.

En général, la prise de conscience pédagogique est beaucoup moins nette qu'à la ville. Et cela écarte quelques faux problèmes : on ne trouve pas de questions angoissées sur l'éducation sexuelle ou sur les "caprices". Mais dans l'ensemble on ne peut se féliciter de 1indifférence aux problèmes que posent les initiations culturelles, artistiques et intellectuelles, le développement de la pensée sociale, de l'esprit critique, de l'esprit de progrès. Les maîtres sont presque seuls à assumer ces tâches, et seuls ne peuvent pas toujours les accomplir de façon satisfaisante ; c'est, d'ailleurs, après 14 ans que beaucoup d'entre elles s'imposent alors que le maître le plus souvent perd de son influence sur ses anciens élèves. Ce n'est pas seulement la prolongation de la scolarité qui est nécessaire, c'est la constitution d'un vrai milieu culturel au village .

On pourrait croire que dans cette éducation agissent deux influences antagonistes ; celle de l'école et celle de la famille. Et cela est vrai pour certains enfants, pour ceux que passionnent les problèmes culturels, et qui, pour la plupart, quittent la campagne pour devenir fonctionnaires. Mais dans l'ensemble des cas c'est la famille qui l'emporte car c'est elle qui fournit les valeurs, les idéaux qui occupent les pensées de l'enfant : travail, production, propriété. C'est en les adoptant que

\footnotetext{
${ }^{5}$ Cf. Th. MAURETTE et H. GRATIOT-ALPHANDÉRY, loc. cit.
} 
l'enfant a conscience de grandir et de devenir un homme, puisqu'il n'y a pas de groupements extra familiaux, sociétés de jeunes, syndicats ou troupes artistiques qui apportent à l'adolescent la conscience qu'il peut remplir une multiplicité de rôles.

Il en résulte une personnalité cohérente et relativement équilibrée mais à la croissance lente et aux aspirations restreintes. Une comparaison avec la formation des enfants à la ville nous permettra de mieux saisir les caractéristiques de l'éducation rurale. 